Valoraciones acerca de la

colaboración en línea en

estudiantes de posgrado en
Assessments about online collaboration in postgraduate students in health services

\title{
servicios de salud
}

José Luis Soto Ortiz* | Carlos Arturo Torres Gastelú** | Juan Carlos Zamudio Hermida***

Recepción del artículo: 26/3/2018 | Aceptación para publicación: 8/6/2018 | Publicación: 30/9/2018

\section{RESUMEN}

En los últimos años, la demanda de personal especializado en los servicios de asistencia en salud se ha incrementado; esto ha propiciado que los profesionales en esta área busquen alternativas de actualización que se adapten a los ritmos y las jornadas laborales de acuerdo con su contratación. El Instituto Universitario Veracruzano ha iniciado la maestría en Administración de los Servicios de Salud en la modalidad en línea en cuyo modelo educativo se encuentran las didácticas de tipo colaborativo. El objetivo de este artículo es analizar las valoraciones acerca de la colaboración en línea en un grupo de 26 estudiantes del primer cuatrimestre de la asignatura en Sistemas y tecnologías de la información. El estudio fue de corte mixto: en lo cuantitativo se aplicó un instrumento denominado Colab, con un índice de confiabilidad de alfa de Cronbach de 0,87, y en lo cualitativo se analizaron las consignas colaborativas realizadas en los foros de discusión. Los resultados señalan una aceptación favorable para este tipo de actividades, por lo que se concluye que los ambientes virtuales de aprendizaje resultan favorables para quienes tienen un horario laboral que les impide estudiar en la modalidad presencial. Asimismo, las didácticas aplicadas en el aprendizaje en línea resultan favorables para el desarrollo de habilidades de colaboración.

\section{Abstract}

In recent years the demand for specialized personnel in health care services has increased, this has encouraged the professionals in this area to look for alternatives of updating that adapt to the rhythms and working days according to their recruitment. The Instituto Universitario Veracruzano has started with the Master's Degree in Health Services in the online modality. The objective was to analyze the assessments on the online collaboration of a group of 26 students of the first semester in the subject in Information Systems and Technologies. The type of study was mixed, for the quantitative part was applied an instrument called "Colab" with a reliability index of Cronbach Alpha of 0.87. For the qualitative part, we analyzed the collaborative slogans made in the discussion forums. The results $o b-$ tained indicates a favorable acceptance for this type of activities. Finally, it is concluded that virtual learning environments are favorable for those subjects whose working hours prevent them from studying in the face-to-face mode. Also, the didactics applied in online learning are favorable for the development of collaboration skills.
Palabras clave

Aprendizaje colaborativo, TIC, educación superior, aprendizaje
Keywords

Collaborative learning, TIC, higher education, learning

* Doctor en Sistemas y Ambientes Educativos. Investigador de El Colegio de Veracruz. Profesor de Asignatura B. Facultad de Estadística e Informática Universidad Veracruzana. Xalapa, Veracruz, México. http://orcid.org/0000-0001-9974-818X. | ** Doctor en Ciencias de la Administración. Profesor-investigador de la Facultad de Contabilidad y Administración, Universidad Veracruzana. SNI-1 Conacyt, Veracruz, Veracurz, México. http://orcid.org/0000-0003-2527-9602. $\mid * * *$ Maestro en Docencia Universitaria. Director de IUVirtual del Instituto Universitario Veracruzano, Banderilla, Veracruz, México. 


\section{INTRODUCCIÓN}

$\mathrm{E}_{\mathrm{r}}^{\mathrm{n}}$ n México resulta imprescindible contar con recursos humanos especializados en salud que cumplan con las expectativas de competencia, calidad y ética (Bárcenas, Ruiz-Velasco, Domínguez y Tolosa, 2015). En los últimos años, la demanda de personal especializado en los servicios de asistencia en salud incrementó; esto ha propiciado que los actores clave en esta área busquen la profesionalización mediante alternativas de actualización que se adapten a los ritmos y las jornadas que demandan sus ámbitos laborales, los cuales dificultan su traslado a las instituciones de formación.

Los profesionales de la salud, como médicos, laboratoristas, enfermeros, han optado por ambientes flexibles de aprendizaje, como el aprendizaje en línea (Margolis, 2012; Moya, 2012). Lo anterior se confirma con lo indicado por Molina, Valencia y Calle (2009), quienes destacan las bondades que ofrece el aprendizaje en línea, como la accesibilidad para aquellos trabajadores con horarios poco flexibles o limitados por las barreras espaciales. Los estudios en línea resultan atractivos para los profesionales que buscan una superación continua, lo que cambia el paradigma de la educación presencial (Harrison, Gemmel \& Reed, 2014).

En la educación de las ciencias médicas presentan un auge los cursos relacionados con los servicios asistenciales de salud, como los que se ofrecen en el marco del proyecto e-Edumed (Avramescu, Marius \& Camen, 2016), cuya actualización de habilidades y competencias se centra en el aprendizaje desde el internet, con materiales de entrenamiento asíncronos y módulos interactivos para la interpretación de los resultados de 
ultrasonido asistidos de forma sincrónica a través de videoconferencias impartidas por tutores expertos en el ramo.

Molina, Valencia y Calle (2009) destacan el creciente uso de la tecnología en salud, lo que ha generado una demanda por parte de médicos para mejorar el conocimiento y la gestión en el área de tecnología biomédica. Sin embargo, los altos costos en el equipamiento médico y la escasez de personal técnico en hospitales producen un impacto en los servicios asistenciales de salud. En este sentido, Molina et al. (2009) diseñaron un diplomado en línea para la gestión y el soporte de equipos médicos impartido a 118 estudiantes peruanos y colombianos, cuyos resultados han sido satisfactorios debido a la aceptación de estos cursos a distancia por parte de los participantes.

Lo anterior ha propiciado un cambio en las pedagogías en el sector salud en relación con el proceso de enseñanza-aprendizaje, que van a la par de los avances tecnológicos. Ejemplo de esto es el estudio de la anatomía humana mediante cursos por internet, en los cuales se enseña la disecación de cadáveres a través de videos o por medio de simuladores, como son los cuerpos artificiales (Harrison, Gemmel \& Redd, 2014).

Los avances tecnológicos han permitido la publicación en internet de contenidos del área de salud en una diversidad de formatos: archivos

\section{Molina, Valencia y Calle (2009)}

\section{destacan el creciente uso de}

\section{la tecnología en salud, lo que} ha generado una demanda por parte de médicos para mejorar el conocimiento y la gestión en el área de tecnología biomédica de texto, multimedia, herramientas interactivas y discusiones virtuales, lo que ha brindado una oportunidad para el aprendizaje en línea. Mendoza (2011) señala que la World Wide Web ha generado pasión por aprender en el medio profesional, el trabajo, el hogar o en lugares de esparcimiento. Sin embargo, esto requiere métodos formales de fácil acceso para el personal que labora en las instituciones públicas de salud (Molina, Valencia y Calle, 2009).

Finalmente, Vázquez-Mata (2008) sostiene que la práctica médica en la actualidad tiene aspectos diferentes que los de hace décadas, entre los cuales resaltan el trabajo en equipo y multidisciplinario, la complejidad de toma de decisiones, las múltiples fuentes de datos para la obtención de información, además de la constante actualización.

La finalidad de este trabajo es analizar las valoraciones por parte de un grupo de estudiantes de la maestría en Administración y Servicios de Salud en la modalidad virtual, que participaron en actividades colaborativas asincrónicas en un aula virtual.

\section{APROXIMACIÓN TEÓRICA CONCEPTUAL}

Los avances tecnológicos permiten la adecuación de ambientes virtuales de aprendizaje (AVA). Una aplicación computacional abre la posibilidad de adaptación de modelos pedagógicos que faciliten la comunicación entre los participantes y la adquisición de conocimientos con soporte de un sitio web. De este modo, a través de un AVA se distribuyen materiales educativos en formato digital (texto, imágenes, audio y video) y se ofrecen programas interactivos como chats, foros de discusión y mensajes que posibilitan la participación e interacción de los integrantes. Además, funciona como un sistema de gestión y soporta el seguimiento y progreso de las actividades realizadas por los participantes. De acuerdo con Boneu (2007), las características principales de un AVA son: 
- Tecnológicas. Este rubro se relaciona con las especificaciones que tiene el aplicativo para la implementación de un AVA respecto al tipo de licencia, si es comercial o código abierto; el soporte del idioma, que debe ser de preferencia compatible con el lenguaje de los participantes; y el sistema operativo y las características del equipo tecnológico que va a soportar la plataforma del AVA.

- Pedagógicas. La mayoría de los sistemas AVA disponen de herramientas y recursos que facilitan la comunicación e interacción entre los usuarios por medio de programas síncronos o asíncronos dentro de la plataforma. Estos permiten la creación de actividades interactivas y la puesta en marcha de las estrategias colaborativas. Asimismo, ofrecen el seguimiento y la evaluación de los estudiantes.

La formación y actualización continua posterior a los estudios de licenciatura se ha beneficiado con el desarrollo de las TIC a través de los AVA; lo anterior contribuye al proceso de formación en cualquier lugar y casi al instante. $\mathrm{Al}$ respecto, $\mathrm{Ca}$ pacho (2011) señala que el nuevo perfil económico requiere una educación renovada y, por lo tanto, el sujeto tiene que ser activo en su proceso de aprendizaje. En este sentido, la formación y actualización virtual juegan un papel importante, ya que, a diferencia del aprendizaje cara a cara, en el cual el sujeto puede o no ser participativo, en el contexto virtual debe ser proactivo al menos para conectarse al servidor, que representa el espacio de aprendizaje. Ahí reside la relevancia de los AVA, los cuales pueden ser adaptativos al estilo de enseñanza. En otras palabras, para el proceso de aprendizaje en los AVA se requiere enfoques psicológicos y pedagógicos que propicien el uso de herramientas flexibles, dinámicas y colaborativas, con contenidos formativos basados en la comunicación sincrónica y asincrónica.

Un AVA está estructurado sistemáticamente para emplearse en los cursos de aprendizaje en línea o a distancia. Un ambiente virtual de aprendizaje
La mayoría de los sistemas AVA disponen de herramientas y recursos que facilitan la comunicación e interacción entre los usuarios

\author{
por medio de programas \\ síncronos o asíncronos dentro
}

de la misma plataforma

es una aplicación de las tecnologías de la información y comunicación diseñada para facilitar la comunicación pedagógica profesor-estudiante en un proceso de enseñanza-aprendizaje que promueve la autoconstrucción del sujeto educable (Bravo, 2012, p. 6).

\section{CENTROS VIRTUALES DE APRENDIZAJE}

Los centros virtuales de aprendizaje están conformados por una diversidad de instituciones educativas que ofrecen, capacitación y formación a través de la red. Estas instituciones pueden ser privadas o públicas y, en su mayoría, se localizan en universidades locales, estatales, nacionales o internacionales con reconocimiento oficial $(\mathrm{Ca}-$ pacho, 2011). También existen instituciones que ofrecen programas sin créditos o certificaciones, como los cursos de actualización o los denominados cursos en línea masivos y abiertos (MOOC, por sus siglas en inglés).

De acuerdo con Capacho (2011), resulta complicado identificar una taxonomía respecto a los términos básicos que tienen que ver con los procesos de formación virtual. Sin embargo, diversos autores han hecho aproximaciones conceptuales 


\section{La educación virtual se concibe} hoy como una modalidad de aprendizaje flexible, que rompe el paradigma de la temporalidad de espacio y tiempo, y fomenta el desarrollo de capacidades para el estudio independiente

de la universidad virtual, la mayoría basadas en el e-learning. Rigo y Ávila (2009) señalan que el e-learning es un enfoque innovador para ofrecer ambientes de aprendizaje con acceso casi en cualquier lugar y en todo tiempo, en el que se utilizan los recursos digitales, así como los materiales apropiados para el aprendizaje de modo ubicuo.

Los estudios de Roldán y Hervas (2008) apuntan que el e-learning suministra los programas educativos mediante sistemas electrónicos y transfiere el conocimiento de tal manera que se convierte en un medio didáctico para que los participantes adquieran habilidades y capacidades que favorezcan su desarrollo profesional.

Monteiro, Leite y Lima (2012) señalan las ventajas del e-learning en educación superior, por ejemplo, el potencial para facilitar los materiales de apoyo, la reducción de los costos de desplazamiento y el impacto medioambiental. El beneficio adicional radica en la flexibilidad de las plataformas de enseñanza que se adaptan a las necesidades de cada institución educativa (Triana, 2016).

Para los procesos de formación virtual, el diseño e implementación de centros educativos virtuales requiere la aplicación de plataformas virtuales, concebidas como herramientas metodológicas para la aplicación del e-learning, las cuales, a su vez, facilitan la adaptación de diversos métodos y didácticas de enseñanza (Díaz y Castro, 2017). De este modo, las plataformas virtuales se conciben, en la actualidad, como importantes herramientas metodológicas para el proceso de formación en espacios virtuales.

En resumen, la formación en espacios virtuales de aprendizaje representa una oportunidad para la actualización de profesionales y obtención de competencias necesarias para la innovación, que rebasan la distancia y logran la pertinencia de escenarios propicios para el desarrollo educativo:

Hoy en día la distancia tiene un significado diferente al que se tenía en el siglo pasado: distancia quiere decir que cada estudiante aprende en su tiempo, que no tiene por qué coincidir con el del resto de compañeros, y lo hace a su ritmo. Distancia quiere decir un tiempo y un espacio imaginario que para todo el mundo es un elemento cotidiano e integrado (Gros, 2011, p. 41).

Por lo tanto, cuando los participantes interactúan en entornos virtuales, la experiencia de su formación se ve enriquecida y facilita el proceso de aprendizaje desde el acceso, adopción, adaptación y apropiación de los recursos y herramientas digitales; estos, de acuerdo con el logro de competencias, serán parte de su entorno en el proceso educativo.

La educación virtual se concibe como una modalidad de aprendizaje flexible, que rompe el paradigma espacio-tiempo, y fomenta el desarrollo de capacidades para el estudio independiente, así como la organización y disposición para el uso de la tecnología. De esta manera, la profesionalización de los actores en los servicios de salud representa un abanico de posibilidades para su desarrollo profesional, ya que, al estar en interacción con otros pares de su sector, experimentan acciones de formación y colaboración en red que forman y transforman su conceptualización del aprendizaje. Así, más allá de las características y bondades del internet en cuanto espacialidad y temporalidad, este propicia las bases para la colaboración en línea en los AVA (Murua, Cacheiro y Gallego, 2014). 


\section{Técnicas y estrategias de colaboración}

Para llevar a cabo la colaboración en línea se requiere el diseño de estrategias didácticas que fomenten las habilidades de tipo social, el trabajo en equipo, la responsabilidad individual y grupal mediante el uso de aplicaciones asíncronas y síncronas, como los foros de debate, chats, intercambio de mensajes y correo (Cheng, Wang, Huang \& Zarifis, 2016; Gutierrez-Porlan, Román-García y Del-Mar, 2018).

Aunado a lo anterior, la colaboración en línea puede desarrollarse mediante las herramientas de tipo colaborativo que permiten lograr los consensos grupales y cuya composición del grupo ha de ser heterogénea y pequeña en número de participantes (no más de cuatro). Asimismo, depende del tipo de tarea: si es fácil, no amerita un trabajo grupal; sin embargo, si la tarea es compleja, esta puede ayudar a alcanzar altos niveles de pensamiento y hacer competitivo tanto al grupo como al alumno (Capacho, 2011).

De este modo, podemos establecer que la colaboración en línea se basa en tres características fundamentales: la interactividad,

\section{Para llevar a cabo la colaboración} en línea se requiere el diseño de didácticas que fomenten las habilidades de tipo social, el trabajo en equipo, la responsabilidad individual y grupal mediante el uso de aplicaciones asíncronas y síncronas la sincronicidad y la negociación. El grado de interacción se puede dar entre pares y no está definido por la frecuencia de interacciones, sino por la forma en que estas pueden influenciar el proceso cognitivo del resto de los miembros del equipo. El segundo elemento es la sincronicidad, término que proviene del campo tecnológico en el que las herramientas de comunicación se definen como sincrónicas o asincrónicas.

Para que se produzca el trabajo colaborativo, son indispensables los intercambios entre pares en las aulas virtuales, producidos a través de los diálogos sostenidos en la participación e interacción en formato textual (Rosario, 2008). Aunado a ello, la interacción posibilita el avance en los aprendizajes, por lo que se requiere la comprensión conjunta del tema que se aborda. Lo anterior implica que los participantes tomen en cuenta lo que su "par" comprende a fin de que todos construyan una visión compartida. Para tener una colaboración efectiva, son necesarias nuevas alternativas que permitan reelaborar ideas para comunicarlas y fundamentar lo que se expone a los demás. Por ello, la interacción resulta importante para la resolución de problemas y el consenso del trabajo colaborativo.

En resumen, las interacciones colaborativas también son negociables y se diferencian por la situación jerárquica; por ejemplo, un participante no impone su punto de vista sobre la base de su autoridad, sino que la negocia argumentando sus razones y perspectivas para tratar de convencer (Collazos y Mendoza, 2006). De ahí que se espere que la estructura de diálogos sea más compleja. Este también es un proceso que se desarrolla gradualmente entre los integrantes de este equipo con base en el concepto de la responsabilidad mutua del aprendizaje de cada uno (Johnson \& Johnson, 1997). 


\section{Estudios relacionados}

En este apartado presentamos algunas investigaciones que dan cuenta de la importancia de los estudios en línea en el sector salud, como las valoraciones en el éxito de estos. Avramescu, Marius y Camen (2016), BarefieldCondon, McCuen y Sayles (2010) y Westbrook (2012) destacan la importancia de la capacitación en el sector salud soportada en AVA. De igual forma, la literatura consultada señala que los cursos en línea mejoran las habilidades para la resolución de problemas, la toma de decisiones y la capacidad del trabajo colaborativo.

En Cuba, la educación a distancia se ha planteado como una solución viable para la capacitación del sector médico, acorde con los tiempos de la sociedad de la información. Rojas, Pérez, Torres y Peláez (2014) formularon una propuesta con el objeto de facilitar el acceso a la información y el conocimiento en el sistema nacional de salud en el sentido de incorporarse a la red Infomed con la intención de fortalecer los procesos de aprendizaje y contribuir al fomento del autoaprendizaje. En la actualidad, esta red es considerada como el espacio interuniversitario para el aprendizaje en línea de la educación médica cubana.

En Rumania, Avramescu, Marius y Camen (2016) desarrollaron un curso en línea para la capacitación de ultrasonido para las enfermeras. La capacitación se llevó a cabo en la plataforma e-Edumed; el contenido educativo estuvo compuesto por seis unidades, que contaron con 126 imágenes, 335 hojas de PowerPoint, 10 clases virtuales y 80 preguntas. Los resultados del estudio revelan que las imágenes en tercera dimensión reforzaron el conocimiento teórico adquirido. Por su parte, la herramienta de los foros facilitó el intercambio de información
En México destaca la oferta

\section{educativa de la Escuela de}

\section{Salud Pública, al igual que}

otras instituciones de educación

\section{superior, como la Universidad}

Nacional Autónoma de México

(UNAM), el Instituto Politécnico

Nacional y el Nodo México del

Campus Virtual de Salud Pública

con los pares y fomentó la comunicación bidireccional, así como la reciprocidad y colaboración entre los participantes.

En el centro virtual de aprendizaje perteneciente al Reino Unido, Harrison, Gemmel y Reed (2014) llevaron a cabo un estudio cuyo objetivo fue evaluar la satisfacción de un grupo de estudiantes de un doctorado en salud pública. Para ello, diseñaron una práctica pedagógica innovadora en la asignatura de Disertación; los doctorandos se prepararon durante doce meses apoyándose en el aula virtual. Asimismo, hicieron uso de las herramientas tecnológicas como los foros y chats. Los resultados apuntan $85 \%$ de satisfacción de la propuesta innovadora.

En México destaca la oferta educativa de la Escuela de Salud Pública, al igual que otras instituciones de educación superior, como la Universidad Nacional Autónoma de México (UNAM), el Instituto Politécnico Nacional y el Nodo México del Campus Virtual de Salud Pública, que alberga a más de veinte instituciones públicas de 
educación y de salud, cuya matrícula se ha incrementado por la demanda de cursos virtuales (Magaña-Valladares, Suárez-Conejero, Hernández-Ávila y Gudiño-Cejudo, 2014).

En la UNAM, Bárcenas et al. (2015) instalaron un laboratorio cibertrónico donde profesoras de nivel licenciatura en enfermería del sector público de salud en México iniciaron su primer contacto con las tecnologías de la información y la comunicación (TIC). El objetivo de la investigación se centró en dos vertientes: adquirir conocimientos básicos en el uso de las TIC y obtener el nivel adecuado para incursionar en programas de formación y actualización docente en línea. Las actividades principales fueron el intercambio de archivos en la plataforma, así como el envío y la recepción de mensajes a través de la plataforma Moodle. El laboratorio empleó recursos para el trabajo colaborativo como chats, videoconferencias y foros. Entre los temas académicos destacaron la tanatología, la prevención, el control de estrés, la diabetes, la geriatría y otros aspectos de la enfermería.

En este trabajo exponemos las perspectivas de la formación virtual desde una visión del profesional en el sector de salud, y tenemos como escenario la maestría en Administración de Sistemas de Salud perteneciente al Instituto Universitario Veracruzano. El objetivo es rescatar las experiencias de la formación virtual de un grupo que cursó el primer cuatrimestre en la asignatura Sistemas y tecnologías de la información.

\section{MATERIALES Y MÉTODO}

El Instituto Universitario Veracruzano ofrece diversos cursos, entre los que destaca la actualización permanente por medio de sus programas académicos de formación continua. En nuestro estudio, seleccionamos el grupo de la maestría en Administración de los Servicios de Salud en la modalidad en línea, y tomamos como escenario la asignatura Sistemas y tecnologías de la información, cuyas didácticas se basan en el aprendizaje individual y colaborativo.

\section{Objetivo}

Analizar las valoraciones acerca de la colaboración en el proceso de formación en línea de los profesionales en los servicios de salud.

\section{Tipo de estudio}

El desarrollo metodológico de esta investigación fue de corte mixto. La técnica de recolección de datos cuantitativos se realizó mediante una encuesta orientada a conocer la valoración por parte de los participantes acerca de las actividades de tipo colaborativo. Para complementar el estudio por la parte cualitativa, efectuamos un análisis de los foros que busca profundizar en la interpretación de los resultados obtenidos acerca de la valoración de la colaboración en línea.

El criterio de la muestra se definió como no probabilístico y determinado a conveniencia por así considerarlo pertinente para el análisis de la colaboración en grupos que deben ser pequeños y relevantes para el estudio (Hernández, Fernández y Baptista, 2010). Esta muestra se conformó por 26 estudiantes.

\section{Instrumentos}

El instrumento aplicado deriva de la línea de investigación relativa a la colaboración en AVA; fue elaborado por Soto y Torres (2016) y se ha aplicado en otros trabajos con el mismo tema. El instrumento suministrado es un cuestionario denominado Colab, el cual se integró de cuatro ítems de identificación personal y 16 ítems agrupados en dos dimensiones (percepción y actitud) para analizar las valoraciones del trabajo colaborativo por parte de los participantes. Utilizamos una escala Likert de cuatro valores: determinante, significativo, poco y nulo, construido acorde con las dimensiones e indicadores (ver figura). 


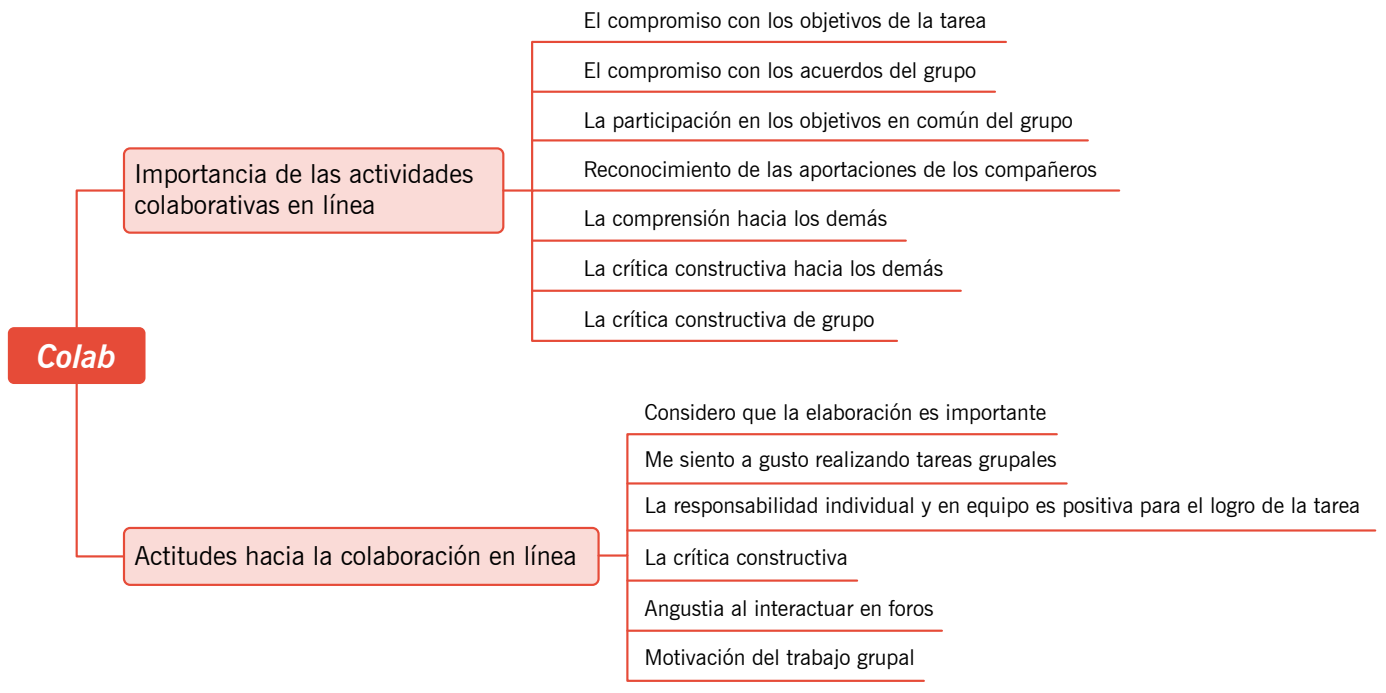

Figura. Dimensiones e indicadores de la colaboración en línea.

\section{Análisis de confiabilidad}

La confiabilidad del instrumento se determinó por medio del coeficiente de alfa de Cronbach, por cada dimensión: percepción hacia la colaboración $(0,823)$ y actitudes hacia la colaboración $(0,831)$, con un coeficiente de Cronbach de o,870 del cuestionario. Los índices de consistencia fueron bastante aceptables y oscilaron entre el intervalo de 0,8 a 1, por lo que son niveles altos y señalan un buen índice de fiabilidad (Bisquerra, 2009). El instrumento se diseñó en Google Forms y se aplicó al final del cuatrimestre; esto permitió conocer las valoraciones respecto a la implementación de la actividad didáctica. Cabe señalar que se les hizo hincapié a los estudiantes que esa encuesta no tendría valoración en sus calificaciones.

En el análisis cualitativo de datos, examinamos el discurso proveniente de los foros de discusión y, para su interpretación, utilizamos la herramienta QDAMinerLite. Este proceso involucró las etapas de preparación de los datos, el análisis inicial y los resultados. Para la preparación de los datos, nos apoyamos en el procedimiento de codificación abierta basado en el estudio de Soto (2014) e incluimos las categorías de interdependencia positiva, habilidades de comunicación e intercambio de información.

\section{Actividades didácticas}

El modelo educativo virtual del Instituto Universitario Veracruzano se basa en el enfoque del constructivismo sociocultural, en el cual intervienen de manera activa los docentes y alumnos en situaciones de trabajo colaborativo que propician la negociación mediante explicaciones, acuerdos y desacuerdos, que, a su vez, estimulan los mecanismos cognitivos a través del espacio virtual para la generación del aprendizaje (Mayorga y Ramos, 2016).

El objetivo final de la asignatura es proporcionar los conocimientos respecto a los sistemas y tecnologías de la información agrupados en cuatro grandes temas que se distribuyen en seis clases: a) bases de los sistemas de información, b) tecnología de la información, c) aplicaciones de la tecnología de la información, y d) administración de la tecnología de la información. En cada tema se incluyeron los objetivos, los materiales 
de consulta, la guía de puntaje, foro para la resolución de dudas y foros de debate, así como el espacio para subir las consignas para la evaluación de cada tema.

Respecto a la evaluación del curso, mediante una rúbrica de evaluación se calificaron los trabajos entregados, la asistencia al aula virtual y la participación en los foros. La descripción de los módulos, la modalidad y la didáctica empleada se presenta en la tabla 1.

La actividad colaborativa se centró en la interacción en un foro de debate que agrupó a los integrantes en equipos de tres o cuatro como máximo. Esta agrupación se integró de manera aleatoria al seleccionar de la lista ordenada de manera alfabética, por ejemplo, el que comenzaba su apellido con la letra " $\mathrm{A}$ ", el intermedio, con letra "M" y el último, con letra " $Z$ ", así sucesivamente hasta conformar los equipos. El espacio de interacción se dio en el foro, que contó con tres momentos: en el primero se les asignó una tecnología (redes, servicios y terminales); para ello debían redactar un texto directamente en el foro y citar todas aquellas actividades didácticas que utilizaran en la institución en la que trabajaban y cómo lo hacían. Asimismo, debían puntualizar las ventajas y desventajas que encuentran al usarlas.

En un segundo momento, debían retomar todas las tecnologías que compartieron y elaborar una presentación en PowerPoint que rescatara los siguientes puntos: tecnología, uso, ventajas y desventajas. Finalmente, en un tercer momento, debían comentar al menos dos presentaciones que hayan llamado su atención y exponer la razón.

\section{RESULTADOS Y DISCUSIÓN}

La información obtenida, de acuerdo con los datos personales, señala que los participantes cursaron una licenciatura relacionada con las ciencias biológicas y de salud. En la gráfica 1 observamos que $37 \%$ corresponde al perfil de enfermería y $17 \%$ a médico general y otro $17 \%$ a médico cirujano.

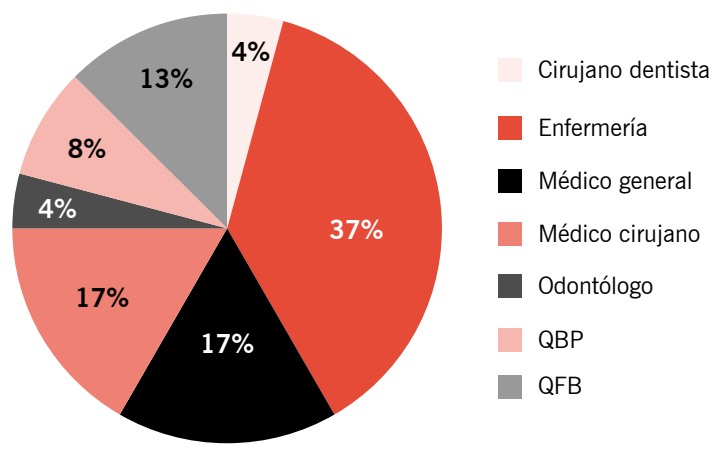

Gráfica 1. Distribución de profesiones del grupo.

Fuente: elaboración propia.

En cuanto a los resultados del instrumento Colab sobre la dimensión de la importancia de las actividades basadas en el trabajo colaborativo, las medias obtenidas señalan que la realización de las consignas e intervención en los foros

Tabla 1. Temas vistos en la asignatura

\begin{tabular}{|c|l|c|l|}
\hline TEMA & \multicolumn{1}{|c|}{ NombRE } & ENFOQUE & DIDÁCTICA EMPLEADA \\
\hline 1 & Bases de los sistemas de información & Individual & $\begin{array}{l}\text { Clase, participación en foros, } \\
\text { entrega de consigna }\end{array}$ \\
\hline 2 & Tecnología de la información & Colaborativa & $\begin{array}{l}\text { Clase, participación en foros, } \\
\text { entrega de consigna }\end{array}$ \\
\hline 3 & Aplicaciones de la tecnología de la información & Colaborativa & Clase, participación en foros \\
\hline 4 & Administración de la tecnología de la información & $\begin{array}{l}\text { Clase, participación en foros, } \\
\text { entrega de proyecto final }\end{array}$ \\
\hline
\end{tabular}

Fuente: elaboración propia. 
fueron aceptables; la media más alta, 3.7200 (ver tabla 2) fue para los indicadores del compromiso con los objetivos de la tarea, el reconocimiento de las aportaciones de los demás y la capacidad de compresión en la resolución de las tareas.

En relación con el análisis cualitativo (ver gráfica 2), se puso en evidencia la dimensión de la interdependencia positiva. Desde el enfoque psicológico, Johnson y Johnson (1997) señalan que la interdependencia positiva es el primero y más importante de los elementos del aprendizaje colaborativo. Requiere el establecimiento de una tarea clara y una meta de grupo para que los estudiantes estén convencidos de que, si uno falla, todos fallan.
Nuestros hallazgos confirman la presencia del indicador intercambio de la información. Al respecto, Johnson y Johnson (1997) aseguran que esta actividad propicia la construcción colectiva del conocimiento. El aspecto central no es que los estudiantes realicen una tarea, sino que logren conjuntamente un aprendizaje significativo.

Lo anterior se ejemplifica con los discursos presentados en los foros de debate: "Al revisar las presentaciones que hasta ahora han compartido en el foro, considero que al tener bibliografía y fuentes de información en común coincidimos en la mayoría de las ventajas y desventajas de cada una de las tecnologías que hemos analizado en nuestras presentaciones" (A12), así como

Tabla 2. Medias obtenidas acerca de la importancia del trabajo colaborativo

\begin{tabular}{|l|c|c|c|}
\hline ¿EN QUÉ GRADO SON IMPORTANTES LOS SIGUIENTES ASPECTOS DEL TRABAJO COLABORATIVO? & MEDIA & DesViación EStándAR & VARIANZA \\
\hline El compromiso con los objetivos de la tarea & 3.7200 & .45826 & .210 \\
\hline El compromiso con los acuerdos del grupo & 3.6000 & .57735 & .333 \\
\hline Participación en los objetivos en común con el grupo & 3.6000 & .57735 & .340 \\
\hline Reconozco las aportaciones de los demás & 3.7200 & .54160 & .210 \\
\hline El cambio de rol me permitió comprender la diversidad de la tarea & 3.5600 & .58310 & .340 \\
\hline Me siento capaz de comprender a los demás en la resolución de tareas & 3.7200 & .5461 & .293 \\
\hline $\begin{array}{l}\text { La crítica constructiva me permite comprender los diferentes puntos } \\
\text { de los demás }\end{array}$ & 3.2800 & .73711 & .543 \\
\hline La crítica constructiva nos permitió mejorar la tarea & 3.5600 & .58310 & .340 \\
\hline
\end{tabular}

Fuente: elaboración propia.

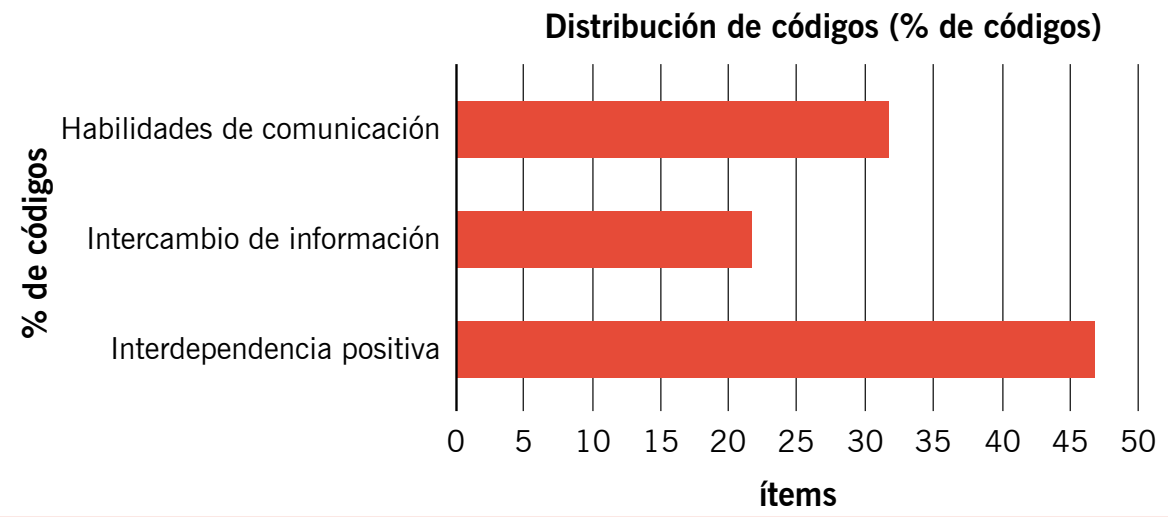

Gráfica 2. Distribución de códigos de la colaboración en línea.

Fuente: elaboración propia. 
la retroalimentación por parte de su compañero: "He revisado tu presentación y al igual que mi compañera y que todos, concordamos en que uno de los bemoles de la tecnología es la inseguridad informática; sin embargo, las ventajas son impresionantes: menos tiempo, más información, más accesibilidad" (A20).

En referencia a la media más baja, 3.2800, acerca de la crítica constructiva realizada por los demás compañeros, indica un nivel medio en cuanto a la aceptación de las críticas y los consejos por parte del grupo. Esto se manifiesta en el comentario de uno de los alumnos:

Buenas tardes, cerrando con mi participación, concluyo que en general cada una de las aportaciones y retroalimentaciones de las presentaciones que observé concuerdan enormemente, y en sus conclusiones todos creemos la importancia que trae consigo la tecnología en nuestra vida y descubrimientos que ayudan a que nuestra esperanza de vida sea más larga con ayuda de nuevos sistemas de tratamiento y diagnóstico que ya existen, entre muchas otras cosas, facilitarnos el día a día tanto dentro del trabajo, hogares y en nuestros vehículos (A6).

En cuanto a las actitudes acerca del trabajo colaborativo en línea conforme a las medias obtenidas (ver tabla 3), la media alta de 3.6800 destaca que los participantes se sienten cómodos al colaborar con sus compañeros. Un participante expone sus apreciaciones acerca del trabajo colaborativo realizado en esta asignatura (A9):

Sería redundante hablar de las bondades y prerrogativas del trabajo colaborativo realizado acerca del tema de las tecnologías, ya que las hemos abordado y acotado de buena manera en este foro, pero a manera de conclusión, creo que la conjunción y empleo de todas ellas en nuestro día a día y en nuestros lugares de trabajo nos permitirán aprovechar al máximo el potencial de estas herramientas sin ser unas más importantes que otras, sino que cada una toma su relevancia en un instante o momento dado y está en nosotros como profesionales el evaluar el momento específico y adecuado de su utilización (A9).

Las segundas medias altas de 3.600 ponen de manifiesto la motivación acerca del trabajo grupal y hacen hincapié en la responsabilidad individual y en equipo para el logro de la tarea. Lo anterior se aprecia en los comentarios de uno de los participantes al señalar también agrado por el aprendizaje virtual: "Hola, compañero. Me gustó tu presentación y me llama la atención todo lo referente a e-learning, que es lo que vivimos en la Universidad Virtual, creo que son acordes la ventajas y desventajas (A3)".

Los estudiantes comentan las aportaciones en los foros:

Concuerdo contigo, la colaboración en el área médica juega un papel súper importante. Por otro lado

Tabla 3. Actitudes acerca del trabajo colaborativo en línea

\begin{tabular}{|l|c|c|c|}
\hline \multicolumn{1}{|c|}{ ActitudeS ACERCA DEL TRABAJO COLABORATIV0 EN LínEA } & MEDIA & DesViaCIÓN ESTáNDAR & VARIANZA \\
\hline $\begin{array}{l}\text { Considero que la colaboración en línea es importante para mi aprendizaje } \\
\text { en el momento actual }\end{array}$ & 3.4400 & .58310 & .340 \\
\hline Me siento a gusto colaborando con mis compañeros & 3.6800 & .55678 & .310 \\
\hline La responsabilidad individual y en equipo es positiva para el logro de la tarea & 3.6000 & .75719 & .573 \\
\hline La crítica en el grupo me ayudó a mejorar mis aportaciones & 3.4000 & .91287 & .833 \\
\hline El interactuar en foros me angustia & 1.4400 & .71181 & .507 \\
\hline Me parece conveniente introducir la colaboración en línea en la enseñanza & 3.4800 & .77028 & .593 \\
\hline Me motiva el trabajo grupal, siempre aprendo de las experiencias de otros & 3.6000 & .50000 & .250 \\
\hline
\end{tabular}

Fuente: elaboración propia. 
la tecnología nos facilita la vida, en cuanto a trámites, consultas, estudios, comunicación, transmisión y recibimiento de información, etcétera. Gracias a la tecnología demostrada en aparatos para estudios, para cirugías, nanotecnología, etcétera, se facilita la atención hacia el derechohabiente, otorgándole una mejor calidad de vida, detección oportuna de ciertos padecimientos e, inclusive, una mejor y más rápida recuperación por ser menos invasivo (A11).

Evidentemente, la colaboración en línea en los foros constituye un espacio para desarrollar estas habilidades en los estudiantes debido a las interacciones en el espacio virtual.

\section{CONCLUSIONES}

La actualización permanente del recurso humano en el sector salud es fundamental; sin embargo, la brecha entre el espacio laboral y el tiempo para tomar cursos o estudiar posgrados difícilmente se acorta en la mayoría de los trabajadores de este sector. Lo anterior se ha visto contrarrestado por los ambientes flexibles de aprendizaje, entre ellos, los cursos en la modalidad virtual, que cada vez tienen mayor auge en México gracias al aprovechamiento de los recursos tecnológicos, por ejemplo, internet como canal de comunicación.

Resulta factible que las instituciones educativas relacionadas con las ciencias de la salud le apuesten a los cursos y posgrados en la modalidad virtual, que promueven habilidades tan necesarias en la actualidad como las colaborativas. Los resultados de este trabajo evidencian la aceptación de las didácticas encaminadas a la colaboración por parte de los participantes. Al propiciar la interacción en los foros de debates, también se ven fortalecidas las habilidades de tipo argumentativo mediante los textos propios y de los compañeros del aula virtual, en los que interpretan, proponen, refutan, cuestionan o están de acuerdo con lo expuesto por sus compañeros; de igual manera, se fomentan las habilidades de comunicación y de tipo social con el intercambio de mensajes cordiales, que someten a consenso las consignas repartidas dentro del grupo para alcanzar el objetivo en común.

Lo anterior coincide con lo expuesto por Elizaphan, Peter, Oboko y Kihoro (2014), en el sentido de que la colaboración en línea en AVA genera el desarrollo colectivo del conocimiento al activar las habilidades de comunicación social, así como la motivación para interactuar con los demás en la construcción del aprendizaje al desarrollar metas comunes.

A manera de conclusión, los espacios virtuales de aprendizaje poco a poco se consolidan como una alternativa flexible para la profesionalización de los trabajadores del sector salud, a quienes, por cuestiones laborales, les resulta difícil acceder a una capacitación de tipo presencial (Taina, Marius \& Camen, 2016). También podemos afirmar que los espacios virtuales de aprendizaje propician la construcción colectiva del conocimiento al presentar didácticas adecuadas para ello, lo que fortalece la habilidad de la colaboración en línea. a

\section{REFERENCIAS BIBLIOGRÁFICAS}

Avramescu, Taina; Marius, Mitrache \& Camen, Adrian. (2016). Care management: On line-based approaches to nurse education in ultrasound imaging. Trabajo presentado en la International Conference e-learning 2016. Funcha, Madeira, Portugal. Recuperado de https://files.eric.ed.gov/fulltext/ED571465.pdf

Bárcenas, Josefina; Ruiz-Velasco, Enrique; Domínguez, Antonio y Tolosa, José. (2015). Espacios en la Web para la formación de enfermeras: un laboratorio cibertrónico 3.0 como plataforma educativa. México: Instituto de Investigaciones sobre la Universidad y la Educación-México, UNAM. Recuperado de http:// recursos. portaleducoas.org/publicaciones/espacios-en-laweb-para-la-formaci-n-de-enfermeras-un-laboratorio-cibertrnico-30-como

Barefield, Amanda; Condon, Jim; McCuen, Charlotte \& Sayles, Nanette. (2010). Incorporating the e-him virtual lab into the health information administration professional practice experience. Journal 
of Asynchronous Learning Networks, vol. 14, núm. 3, pp. 6873. Recuperado de https://www.learntechlib.org/p/53540/

Bisquerra, Rafael (coord.). (2009). Metodología de la investigación educativa ( $2^{\mathrm{a}}$ ed.). Madrid, España: La Muralla.

Boneu, Josep M. (2007). Plataformas abiertas de e-learning para el soporte de contenidos educativos abiertos. Revista de Universidad y Sociedad del Conocimiento, vol. 4, núm. 1, pp. 36-47. Recuperado de: http://rusc.uoc.edu/rusc/ca/index.php/rusc/ article/download/v4n1-boneu/298-1215-2-PB.pdf

Bravo, M. (2012). Enseñanza-aprendizaje de las matemáticas utilizando como apoyo en ambientes virtuales de aprendizaje. En Y. Sandoval (comp.) J. J. Serrano (ed.)... Las tecnologías de la información en contextos educativos: nuevos escenarios de aprendizaje. (pp.177-202). Cali: Editorial Universidad Santiago de Cali,

Capacho, Jospe Rafael. (2011). Evaluación del aprendizaje en espacios virtuales-TIC. Bogotá, Colombia: ECOE Ediciones.

Cheng, Xusen; Wang, Xueyin; Huang, Jianqing \& Zafiris, Alex. (2016). An Experimental study of satisfaction response: Evaluation of online collaborative learning. International Review of Research in Open and Distributed Learning. Vol. 17, núm. 1, pp. 60-78. Recuperado de: http://www.irrodl.org/index.php/irrodl/article/ view/2110/3607

Collazos, César Alberto y Mendoza, Jair. (2006). Cómo aprovechar el "aprendizaje colaborativo" en el aula. Educación y Educadores, vol. 9, núm. 2, pp. 61-67. Recuperado de http:// www.scielo.org.co/scielo.php?script =sci_abstract\&pid $=$ S0123-12942006000200006

Díaz, Frank y Castro, Ariel. (2017). Requerimientos pedagógicos para un ambiente virtual de aprendizaje. Revista Cubana de Contabilidad y Finanzas COFIN Habana, vol. 11, núm. 1, pp. 1-13. Recuperado de http://scielo.sld.cu/scielo.php?script=sci abst ract\&pid $=$ \$2073-60612017000100004

Elizaphan, Marina; Peter, Waiganjo; Oboko, Robert \& Kihoro, John. (2014). Student's perceived challenges in an online collaborative learning environment: A case of Higher Learning Institutions in Nairobi, Kenya. The International Review of Research in Open and Distributed Learning. Vol. 15, núm. 6, pp. 132-161. http://dx.doi.org/10.19173/irrodl.v15i6.1768

Gros, Begoña. (2011). Evolución y retos de la Educación Virtual. Construyendo el e-learning del Siglo XXI. Barcelona, España: Editorial UOC.

Harrison, Roger; Gemmell, Isla \& Reed, K. (2014). Student satisfaction with a web-based dissertation course: Findings from an
International Distance Learning Master's Programme in Public Health. The International Review of Research in Open and Distance Learning. Vol. 15, núm. 1, pp. 182-202. http://dx.doi. org/10.19173/irrodl.v15i1.1665

Hernández, Roberto; Fernández, Carlos y Baptista, M. Pilar. (2010). Metodología de la Investigación ( $5^{\mathrm{a}}$ ed.). México, DF: McGraw Hill Educación.

Johnson, Frank \& Johnson, David. (1997). Joining together. Group theory and group skils. Boston, EUA: Allyn and Bacon.

Magaña-Valladares, L.; Suárez-Conejero, J.; Hernández-Ávila, N. y Gudiño-Cejudo, M. (2014). La Escuela de Salud Pública de México: innovación educativa y tecnológica en el nuevo milenio. Revista de Salud Pública de México, vol. 56, núm. 6, pp. 660-665. Recuperado de http:// www.scielo.org.mx/scielo.php?script=sci_arttext\&pid $=$ S0036-36342014000600017

Margolis, Álvaro. (2012). Tendencias en educación médica continua a distancia. Revista de Investigación en Educación Médica, vol. 2, núm. 1, pp. 50-54. Recuperado de http://riem. facmed.unam.mx/sites/all/archivos/N2Num01/08_AR_MARGOLIS.PDF

Mayer, R. (2000). Diseño educativo para un aprendizaje constructivista. En Ch. Reigeluth, (eds.). Diseño de la instrucción. Teorías y modelos. (pp. 153-172). Madrid: Ediciones Aula XXI. Santillana.

Mayorga, A. (2016). Generalidades de la tutoría en el modelo de la IUV Universidad Virtual. Veracruz, México: Instituto Universitario Veracruzano.

Mendoza, Enrique. (2011). Aprendizaje electrónico: su papel en la educación médica continua. Revista Pediatría de México, vol. 13, núm. 3, pp. 127-133. Recuperado de http://www.medigraphic.com/pdfs/conapeme/pm-2011/pm113h.pdf

Molina, Tatiana; Valencia, Juan Esteban y Calle, Catalina. (2009). Diseño, desarrollo e implementación de un diplomado en línea en gestión y soporte de equipos médicos. Revista CES Medicina, vol. 23, núm. 2, pp. 73-79. Recuperado de http://revistas.ces. edu.co/index.php/medicina/article/view/912/599

Monteiro, A.; Leite, C. \& Lima, L. (2012). Ensinar e aprender com tecnologias digitais no Ensino Superior. En J. A. Moreira \& A. Monteiro (eds.). Ensinar e Aprender Online com Tecnologias Digitais (pp. 31-44). Porto: Porto Editora.

Moya, Fabio. (2012). Importancia de las TIC en la enseñanza de salud. Revista Coruniamericana, vol. 1, núm. 1, pp. 81-86. Recuperado de http://coruniamericana.edu.co/publicaciones/ojs/ index.php/IID/article/viewFile/175/169. 
Rigo, Marco y Ávila, José Luis. (2009). Ambientes virtuales de aprendizaje y educación superior: una experiencia semipresencial enseñando metodología de investigación educativa. Trabajo presentado en el X Congreso Nacional de Investigación Educativa. México. Recuperado de http://www.comie.org.mx/ congreso/memoriaelectronica/v10/pdf/area_tematica_07/ ponencias/1195-F.pdf

Rojas, Nictadys; Pérez, Flora; Torres, Isbeti y Peláez, Everardo. (2014). Las aulas virtuales: una opción para el desarrollo de la educación médica. Edumecentro, vol. 6, núm. 2, pp. 231-247. Recuperado de http://scielo.sld.cu/scielo.php?script=sci_artt ext\&pid =\$2077-28742014000200016

Rosario, H. (2008). La web. Herramienta de trabajo colaborativo. "Experiencia en la Universidad de Carabobo". Revista de Medios y Educación Pixel-Bit, núm. 31, pp. 131-139.

Soto, J. y Torres, C. (2016). Percepciones y expectativas del aprendizaje en jóvenes universitarios. Revista de Docencia Universitaria. vol. 14, núm. 1, pp. 51-67. https://doi.org/10.4995/ redu.2016.5797
Soto, José Luis. (2014). Desarrollo de la competencia de colaboración en línea. Estudio de casos: Virtu@mi. (Tesis de doctorado.) Universidad Veracruzana, Veracruz, México. Recuperado de https://cdigital.uv.mx/bitstream/handle/123456789/41452/ Soto0rtizJoseLuis.pdf;jsessionid =3B4787FA37991B2E0DA90 OF27C793D5F? sequence $=2$

Triana, E. (2016). Las comunidades virtuales de aprendizaje. Trabajo presentado en el III Congreso Internacional AmITIC2016, Santa Cruz de la Sierra, Bolivia, 27-30 de septiembre.

Vásquez-Mata, G. (2008). Realidad virtual y simulación en el entrenamiento de los estudiantes de Medicina. Revista Educación Médica, vol. 11, supl. 1.

Vygotsky, Lev. (1987). Historia de las funciones psíquicas superiores. La Habana, Cuba: Editorial Científico Técnica.

Westbrook, Catherine. (2012). Online collaborative learning in health care education. European Journal of Open Distance and E-learning. Vol. 1, núm. 23, pp 1-6. Recuperado de http:// www.eurodl.org/index.php

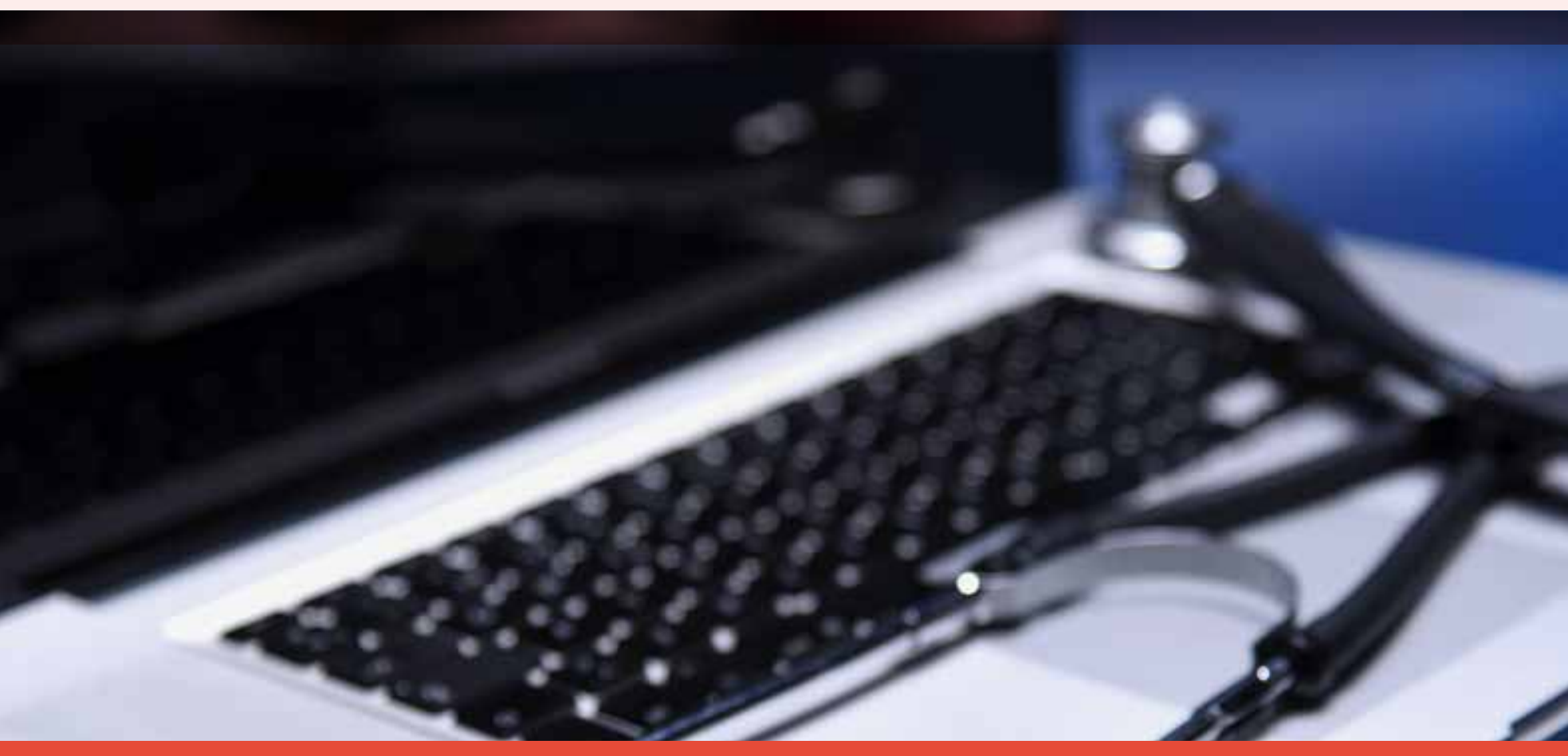

"Este artículo es de acceso abierto. Los usuarios pueden leer, descargar, distribuir, imprimir y enlazar al texto completo, siempre y cuando sea sin fines de lucro y se cite la fuente."

\section{CÓMIO CITAR ESTE ARTÍCULO:}

Soto Ortiz, José Luis; Torres Gastelú, Carlos Arturo y Zamudio Hermida, Juan Carlos. (2018). Valoraciones acerca de la colaboración en línea en estudiantes de posgrado en servicios de salud. Apertura, 10 (2), pp. 64-79. http://dx.doi.org/10.32870/Ap.v10n2.1370 